\title{
NO way! Nasal nitric oxide measurement in infants
}

\author{
Jane S. Lucas $\mathbb{1}^{1,2}$ and Woolf T. Walker ${ }^{1,2}$ \\ Affiliations: ${ }^{1}$ Primary Ciliary Dyskinesia Centre, NIHR Biomedical Research Centre, University Hospital \\ Southampton NHS Foundation Trust, Southampton, UK. ${ }^{2}$ University of Southampton Faculty of Medicine, \\ Academic Unit of Clinical and Experimental Medicine, Southampton, UK.
}

Correspondence: Jane S. Lucas, Southampton University Hospital, Mailpoint 803 F level, Tremona Road, Southampton, S016 6YD, UK. E-mail: jlucas1dasoton.ac.uk

@ERSpublications

Nasal nitric oxide (NO) measurement is feasible in infants, potentially allowing earlier diagnosis of primary ciliary dyskinesia http://ow.ly/9K0a30kpcZU

Cite this article as: Lucas JS, Walker WT. NO way! Nasal nitric oxide measurement in infants. Eur Respir J 2018; 51: 1800958 [https://doi.org/10.1183/13993003.00958-2018].

Primary ciliary dyskinesia (PCD) generally causes symptoms from the first weeks of life, but diagnosis is usually delayed for years or can be missed altogether. Referral for diagnostic testing is often late because key symptoms, such as wet cough, chronic rhinitis and recurrent upper and lower respiratory tract infection, are non-specific. An international survey of patients reported that $37 \%$ had $>40$ visits to medical professionals due to PCD-related symptoms before being referred for diagnostic testing [1]. Approximately 50\% of children with PCD have situs inversus, a rare condition in the general population, and it is therefore not surprising that these patients are investigated earlier. A European survey found that age of diagnosis was at 3.5 years in those with situs inversus and 5.8 years in children with normal situs [2]. Assuming a prevalence of 1 in 10000 [3], we expect $\sim 1180$ diagnosed children in England (childhood population 11.8 million); however, there are currently only $\sim 340$ children in the National Paediatric PCD Service. This highlights a huge proportion of undiagnosed patients even in a country with a national PCD service [4]. This is in stark contrast to cystic fibrosis (CF) where neonatal screening programmes ensure that diagnosis occurs in early infancy in many countries, with positive impact on clinical outcome [5]. Young children with PCD have similarly impaired forced expiratory volume in $1 \mathrm{~s}$ to children with CF [6], and require multidisciplinary treatment. Early diagnosis facilitates a management plan, including regular airway clearance physiotherapy, surveillance for airway pathogens with treatment of respiratory exacerbations, genetic counselling for families, audiology monitoring and management of conductive hearing loss $[7,8]$. We expect that early diagnosis would delay respiratory decline and ensure specialist treatment of ear, hearing and nasal issues, thereby optimising health, psychological, social and educational outcomes.

\section{Which infants should be tested for PCD?}

There is no single gold standard test for PCD diagnosis. Presently, the diagnosis is made in patients with a compatible medical history following a demanding combination of tests including nasal nitric oxide (nNO), high-speed video microscopy, transmission electron microscopy, genetics, and culture of respiratory epithelial cells [7, 9-12]. These tests are costly and need experienced staff using sophisticated equipment, hence testing is restricted to reference centres which may be geographically distant from the patient. Identifying the correct patients for testing is therefore important and screening tools are needed. Although individual symptoms are non-specific, the pattern of symptoms is often characteristic. A typical history might include neonatal respiratory distress in an infant with no risk factors, a daily wet cough starting in early infancy, recurrent chest infections, persistent rhinitis and serous otitis media. Screening 
tools (e.g. PICADAR) to identify patients based on their clinical history are helpful but require a history that might not yet have evolved in young infants $[13,14]$.

\section{$\mathrm{nNO}$ as a diagnostic test for PCD in infancy}

LUNDBERG et al. [15] first reported extremely low nNO concentrations in patients with PCD >20 years ago. A more recent meta-analysis of 11 studies reported a mean \pm SD $\mathrm{nNO}$ output of $19 \pm 18.6 \mathrm{~nL} \cdot \mathrm{min}^{-1}$ in PCD $(\mathrm{n}=478)$ and $265 \pm 118.9 \mathrm{~nL} \cdot \mathrm{min}^{-1}$ in healthy controls $(\mathrm{n}=338)$ [16]. Whilst the reason for low $\mathrm{nNO}$ remains elusive [17], measurement of $\mathrm{nNO}$ has been widely adopted as part of the diagnostic work-up $[9,10,18-23]$. The "gold standard" measurement is made during a velum closure manoeuvre to reduce contamination of nasal gas with lower airway air [24]. This breathing manoeuvre is not possible in young children because it requires patient cooperation to exhale against resistance or to phonate to close the velum during sampling. Hence, this method is not possible in the age group where testing should ideally occur. A number of studies have therefore investigated measurement during tidal mouth breathing and have reported that it provides an acceptable alternative for children who cannot manage velum closure manoeuvres $[18,20,22,23,25]$. However, guidelines have previously suggested that $\mathrm{nNO}$ cannot be used as a test in infancy because of feasibility issues, and because nNO concentrations are very low in healthy infants. This dogma has been challenged by a cross-sectional study of $\mathrm{nNO}$ in infants <1 year [26], and now a study which has longitudinally measured levels throughout infancy from 2 weeks to 2 years [27].

In this issue of the European Respiratory Journal, MARTHIN et al. [27] report the natural evolution of nNO levels in 44 healthy infants who were followed at six time-points from 2 weeks to 2 years. They additionally use retrospective data from infants with PCD. Theirs is the first manuscript to demonstrate the longitudinal increase in nNO over the first years of life. The authors demonstrated that measurement of nNO during tidal breathing is feasible in healthy infants and those with PCD. Importantly, they report that an extremely good success rate is possible (>99\%) [27]. The authors confirmed that success in measuring infant nNO depended on being very patient (personal communication). Newborn infants were apparently easy to measure when fed and asleep. The most challenging ages were 4 to 12 -month olds, who often woke and required comforting by pacifier, parents singing to the child, or distracting with a toy. With 1-2 year olds, a smart phone worked well for distraction (personal communication, J.K. Marthin).

Some healthy infants had very low levels of nNO [27], indistinguishable from PCD, and further PCD diagnostic testing, including a repeat nNO measurement after a couple of months, should be conducted. In particular, healthy infants had reduced nNO during upper respiratory tract infections, highlighting that measurements should only be conducted for PCD diagnostics when infection-free.

\section{Notes of caution}

The study reported by MARTHIN et al. [27] has the potential to change practice at our PCD centre, but further research is needed before widespread testing of infants is recommended. Until now we have been reluctant to recommend $\mathrm{nNO}$ as a test in this age group because we had concerns that taking measurements would be difficult. Now that we know that measurement is feasible, nNO needs evaluating with infants in a diagnostic setting to see whether it can distinguish PCD cases from children with similar symptoms who do not have PCD.

Importantly, to avoid a large number of false-positive cases, rigorous patient selection is needed before applying diagnostic tests. If the test becomes used as a general screening test, the number of false-positive cases would be very high (poor positive predictive value), overwhelming diagnostic services [28, 29]. Classical clinical features in infancy that should prompt testing for PCD include: unexplained neonatal respiratory distress starting a few hours after delivery, often associated with atelectasis/lobar collapse and prolonged oxygen requirement; persistent rhinitis; daily wet cough; and situs inversus or situs ambiguous, with or without congenital heart disease $[18,30]$. However, some infants with PCD may not present as neonates, or their symptoms might be mild; and 50\% of infants with PCD have normal situs which should not delay testing in a symptomatic baby.

Additionally, non-specialist centres need training to conduct the measurements and interpret the results. Local testing might utilise portable electrochemical analysers rather than "gold standard" chemoluminescence analysers; a newly "Conformité Européene" (CE)-marked portable nNO analyser is an improvement on previous portable models, with an $\mathrm{nNO}$ trace allowing the technician to visualise peak nNO readings [31]. As portable NO analysers become more widespread for use in asthma, the opportunity for nNO measurements of infants at centres without PCD diagnostics is potentially useful, but only if measured by technicians who have received training, in correctly selected patients. Importantly, infants with a strong likelihood should be referred to a specialist centre for further diagnostic tests, even if nNO is within normal limits. 


\section{Future perspectives}

There are currently no guidelines to standardise tidal breathing measurements of $\mathrm{nNO}$ and these are now needed. A study from North America demonstrated that measurement during velum closure could be standardised for testing children $>5$ years [19]. By this age structural lung damage may already be present and we need tests which target early diagnosis. MARTHIN et al. [27] have demonstrated that tidal manoeuvres are feasible with potential to contribute to the diagnostic portfolio from the first weeks of life. The tidal breathing method now needs validating in a diagnostic setting because the test might fare less well with disease controls rather than healthy controls [29]. This would need a multicentre approach to include sufficient infants suspected of PCD. Research and consensus will be needed to decide how to take the reading. Marthin et al. [27] used the averaged nNO value from the three highest, distinct, visible peak concentrations measured within 30 to $40 \mathrm{~s}$, and read directly as point values on the screen, whilst other authors have taken the measure from five peaks [25]. Details of quality assessment of the tracings will be an important consideration for standardisation.

\section{Summary}

MARTHIN et al. [27] performed $224 \mathrm{nNO}$ measurements in 44 healthy infants. They reported median (interquartile range) concentrations of 15.0 (9.6 to 22.8) $\mathrm{nL} \cdot \mathrm{min}^{-1}$ in newborns, increasing to 93.4 (67.0 to 128.4$) \mathrm{nL} \cdot \mathrm{min}^{-1}$ at the age of 24 months. Respiratory tract infections temporarily suppressed nNO by $79 \%$ [27]. The success rate of acceptable nNO-sampling was 223/224 (99.6\%). With further validation and standardisation, nNO may prove an important diagnostic tool for infants with PCD. This would allow earlier initiation of appropriate management strategies, thereby reducing the risk of early lung damage and respiratory decline.

Conflict of interest: J.S. Lucas is on the PCD advisory board of Aerocrine/Circassia and has received expenses to attend meetings, research monies and honoraria, outside the submitted work.

\section{References}

1 Behan L, Dunn Galvin A, Rubbo B, et al. Diagnosing primary ciliary dyskinesia: an international patient perspective. Eur Respir J 2016; 48: 1096-1107.

2 Kuehni CE, Frischer T, Strippoli MP, et al. Factors influencing age at diagnosis of primary ciliary dyskinesia in European children. Eur Respir J 2010; 36: 1248-1258.

3 Lucas JS, Walker WT, Kuehni CE, et al. Primary ciliary dyskinesia. In: Cordier J-F, ed. Orphan Lung Diseases (ERS Monograph). Sheffield, ERS, 2011; pp. 1-19.

4 Lucas JS, Chetcuti P, Copeland F, et al. Overcoming challenges in the management of primary ciliary dyskinesia: the UK model. Paediatr Respir Rev 2014; 15: 142-145.

5 Castellani C, Massie J, Sontag M, et al. Newborn screening for cystic fibrosis. Lancet Respir Med 2016; 4: 653-661.

6 Halbeisen F, Goutak M, Maurer E, et al. Lung growth in children and young adults with primary ciliary dyskinesia (PCD): an iPCD cohort study. Eur Respir J 2016; 48: Suppl. 60, PA375.

7 Kuehni CE, Lucas JS. Diagnosis of primary ciliary dyskinesia: summary of the ERS Task Force report. Breathe 2017; 13: 166-178.

8 Lucas JS, Alanin MC, Collins S, et al. Clinical care of children with primary ciliary dyskinesia. Expert Rev Respir Med 2017; 11: 779-790.

9 Shapiro AJ, Zariwala MA, Ferkol T, et al. Diagnosis, monitoring, and treatment of primary ciliary dyskinesia: PCD foundation consensus recommendations based on state of the art review. Ped Pulm 2016; 51: 115-132.

10 Jackson CL, Behan L, Collins SA, et al. Accuracy of diagnostic testing in primary ciliary dyskinesia. Eur Respir J 2016; 47: 837-848

11 Hirst RA, Jackson CL, Coles JL, et al. Culture of primary ciliary dyskinesia epithelial cells at air-liquid interface can alter ciliary phenotype but remains a robust and informative diagnostic aid. PloS One 2014; 9: e89675.

12 Stannard WA, Chilvers MA, Rutman AR, et al. Diagnostic testing of patients suspected of primary ciliary dyskinesia. Am J Respir Crit Care Med 2010; 181: 307-314.

13 Behan L, Dimitrov BD, Kuehni CE, et al. PICADAR: a diagnostic predictive tool for primary ciliary dyskinesia. Eur Respir J 2016; 47: 1103-1112.

14 Leigh MW, Ferkol TW, Davis SD, et al. Clinical features and associated likelihood of primary ciliary dyskinesia in children and adolescents. Ann Am Thorac Soc 2016; 13: 1305-1313.

15 Lundberg JO, Weitzberg E, Nordvall SL, et al. Primarily nasal origin of exhaled nitric oxide and absence in Kartagener's syndrome. Eur Respir J 1994; 7: 1501-1504.

16 Collins SA, Gove K, Walker W, et al. Nasal nitric oxide screening for primary ciliary dyskinesia: systematic review and meta-analysis. Eur Respir J 2014; 44: 1589-1599.

17 Walker WT, Jackson CL, Lackie PM, et al. Nitric oxide in primary ciliary dyskinesia. Eur Respir J 2012; 40: 1024-1032.

18 Lucas JS, Barbato A, Collins SA, et al. European Respiratory Society guidelines for the diagnosis of primary ciliary dyskinesia. Eur Respir J 2017; 49: 1601090.

19 Leigh MW, Hazucha MJ, Chawla KK, et al. Standardizing nasal nitric oxide measurement as a test for primary ciliary dyskinesia. Ann Am Thorac Soc 2013; 10: 574-581.

20 Marthin JK, Nielsen KG. Hand-held tidal breathing nasal nitric oxide measurement--a promising targeted case-finding tool for the diagnosis of primary ciliary dyskinesia. PloS One 2013; 8: e57262.

21 Marthin JK, Nielsen KG. Choice of nasal nitric oxide technique as first-line test for primary ciliary dyskinesia. Eur Respir J 2011; 37: 559-565. 
22 Mateos-Corral D, Coombs R, Grasemann H, et al. Diagnostic value of nasal nitric oxide measured with non-velum closure techniques for children with primary ciliary dyskinesia. J Pediatr 2011; 159: 420-424.

23 Harris A, Bhullar E, Gove K, et al. Validation of a portable nitric oxide analyzer for screening in primary ciliary dyskinesias. BMC Pulm Med 2014; 14: 18.

24 ATS/ERS recommendations for standardized procedures for the online and offline measurement of exhaled lower respiratory nitric oxide and nasal nitric oxide, 2005. Am J Respir Crit Care Med 2005; 171: 912-930.

25 Beydon N, Chambellan A, Alberti C, et al. Technical and practical issues for tidal breathing measurements of nasal nitric oxide in children. Ped Pulm 2015; 50: 1374-1382.

26 Adams PS, Tian X, Zahid M, et al. Establishing normative nasal nitric oxide values in infants. Respir Med 2015; 109: 1126-1130.

27 Marthin JK, Philipsen MC, Rosthoj S, et al. Infant nasal nitric oxide over time: natural evolution and impact of respiratory tract infection. Eur Respir J 2018; 51: 1702503.

28 Collins SA, Behan L, Harris A, et al. The dangers of widespread nitric oxide screening for primary ciliary dyskinesia. Thorax 2016; 71: 560-561.

29 Kuehni CE, Lucas JS. Toward an earlier diagnosis of primary ciliary dyskinesia. Which patients should undergo detailed diagnostic testing? Ann Am Thorac Soc 2016; 13: 1239-1243.

30 Mullowney T, Manson D, Kim R, et al. Primary ciliary dyskinesia and neonatal respiratory distress. Pediatrics 2014; 134: 1160-1166.

31 Lucas J, Leigh M, Beydon $\mathrm{N}$, et al. New portable nasal nitric oxide (nNO) analyser differentiates primary ciliary dyskinesia (PCD) from healthy individuals. Eur Respir J 2017; 50: Suppl. 61, PA1848. 